\title{
Interaction of Kupffer Cells and Platelets Determines the Severity of Ischemia-Reperfusion Injury in Steatosis
}

\author{
Koichi Ogawa, ${ }^{1}$ Tadashi Kondo, ${ }^{1}$ Takafumi Tamura, ${ }^{1}$ Hideki Matsumura, ${ }^{1}$ \\ Kiyoshi Fukunaga, ${ }^{1}$ Soichiro Murata ${ }^{1}$ and Nobuhiro Ohkohchi ${ }^{1}$ \\ ${ }^{1}$ Department of Surgery, Graduate School of Comprehensive Human Sciences, University of Tsukuba, Tsukuba, \\ Ibaraki, Japan
}

Liver steatosis increases the risk of postoperative complications following major liver resection, since the steatotic liver is susceptible to ischemia-reperfusion (IR) injury. However, it is unclear how IR injury changes in relation to the degree of hepatic steatosis. Previously, we reported that interaction between Kupffer cells (KCs) and platelets induced hepatic IR injury. The aim of our present study was to evaluate the relationship between the degree of liver steatosis and IR injury by focusing on the interaction of KCs and platelets. Mild and moderate steatotic liver models were generated in Wistar rats by feeding a cholinedeficient diet for 2 and 4 weeks, respectively. The intensity of steatosis was defined based on the proportion of hepatocytes with fatty infiltration: normal (less than $5 \%$ ), a mild steatosis (5-30\%), and moderate steatosis $(30-60 \%)$. All groups were subjected to $20 \mathrm{~min}$ of warm ischemia followed by $120 \mathrm{~min}$ of reperfusion. The number of adhesion of KCs to platelets in sinusoids was observed by intravital microscopy. IR injury was evaluated with serum alanine aminotransferase levels, histological findings, and sinusoidal perfusion. Compared to the normal liver, mild steatosis reduced the adhesion of KCs to platelets, inducing the attenuation of IR injury. In contrast, moderate steatosis increased the adhesion of $\mathrm{KCs}$ to platelets, aggravating IR injury relative to the normal liver. IR injury in the steatotic liver was not simply proportional to the degree of steatosis. Mild steatosis ameliorates IR injury compared to the normal liver, whereas moderate steatosis increases IR injury.

Keywords: intravital microscopy; ischemia-reperfusion; Kupffer cell; platelet; steatosis Tohoku J. Exp. Med., 2014 February, 232 (2), 105-113. C 2014 Tohoku University Medical Press

\section{Introduction}

Liver steatosis is a risk factor for postoperative complications after liver surgery and liver transplantation (Adam et al. 1991; McCormack et al. 2007). Ischemiareperfusion (IR) injury is one of the most critical complications commonly associated with liver surgery (Delva et al. 1989; Hui et al. 1994). Fatty accumulation in the cytoplasm of hepatocytes is associated with increase in the cell volume, which may induce partial or complete obstruction of the hepatic sinusoid space, so that, as a result of impaired hepatic microcirculation, the steatotic liver has reduced tolerance against IR injury (Ijaz et al. 2003). Steatosis of the liver is classified into three grades according to the proportion of hepatocytes with fatty infiltration: mild $(<30 \%)$, moderate (30-60\%), and severe $(>60 \%$ ) (Adam et al. 1991). It has been shown that the degree of steatosis correlates with higher post-operative liver enzymes and increased long-term mortality of liver transplantation (Perez-Daga et al. 2006). However, no reports have evalu- ated IR injury at different degrees of steatosis in the same experimental models.

Recently, we reported that using intravital microscopy (IVM) we observed that interaction, such as adhesion, between Kupffer cells (KCs) and platelets played a pivotal role in the early period of hepatic IR injury, which occurred within 120 min after reperfusion (Tamura et al. 2012). We also confirmed that interaction between $\mathrm{KCs}$ and platelets correlated with the number of KCs in the hepatic sinusoids (Ogawa et al. 2013). In the present study, we investigated the change in IR injury in mild and moderate steatotic livers compared with the normal liver, focusing on the interaction between KCs and platelets and the number of KCs.

$\mathrm{KCs}$ are important in the inflammatory reaction of the liver (Deng et al. 2009). In addition, there are several reports regarding the association between fatty acids and the inflammatory response (Matsuzaka et al. 2007; Akazawa et al. 2010). Hence we focused on the composition of fatty acids as a factor of changes in $\mathrm{KC}$ numbers. In previous studies, palmitoleate, a monounsaturated fatty acid, was

Received December 5, 2013; revised and accepted January 27, 2014. Published online February 19, 2014; doi: 10.1620/tjem.232.105. Correspondence: Nobuhiro Ohkohchi, M.D, Ph.D., Department of Surgery, Doctoral Program in Clinical Science, Graduate School of Comprehensive Human Sciences, University of Tsukuba, 1-1-1 Tennodai, Tsukuba 305-8575, Japan.

e-mail: nokochi3@md.tsukuba.ac.jp 
shown to increase steatosis but reduce the number of KCs, and decrease inflammatory signaling in the c-Jun $\mathrm{N}$-terminal kinase (JNK) pathway (Akazawa et al. 2010; Guo et al. 2012). In this study, we also evaluated the change in palmitoleate levels and activation of JNK.

\section{Materials and Methods}

Animals

Male Wistar rats were obtained from CLEA Japan, Inc. (Tokyo, Japan). We prepared three model types, normal liver, mild steatosis, and moderate steatosis. We used a choline-deficient diet (CDD) (Oriental Bio Service Kanto Inc., Ibaraki, Japan) to introduce steatosis of the liver. The CDD-induced steatotic liver is an established experimental model, in which morphological and functional features are very similar to those of the clinical steatotic liver (Hayashi et al. 1993). In the normal liver model, rats weighting 250 to $300 \mathrm{~g}$ were used and they were fed a normal chow. In the steatosis models, the rats' weights were adjusted to the same weight range after they were fed a CDD for 2 or 4 weeks. Our criterion to differentiate the intensity of steatosis was the proportion of hepatocytes with fatty infiltration histologically. Thus, we defined the normal liver as having less than $5 \%$, mild steatosis as $5-30 \%$, and moderate steatosis as $30-60 \%$. Animal experiments were carried out in a humane manner after receiving approval from the Institutional University Experiment Committee of the University of Tsukuba, and in accordance with the Regulation for Animal Experiments in our university and the Fundamental Guideline for Proper Conduct of Animal Experiment and Related Activities in Academic Research Institutions under the jurisdiction of the Japanese Ministry of Education, Culture, Sports, Science, and Technology.

\section{Experimental Design}

Animals were divided into three groups: (1) normal liver group $(n=6)$; (2) mild steatosis group $(n=6)$; (3) moderate steatosis group $(n=6)$. In all groups, total normothermic hepatic ischemia was induced for $20 \mathrm{~min}$ by clamping the portal triad (the hepatic artery, portal vein, and bile duct). The hepatic microcirculation and dynamics of platelets and KCs were observed just before ischemia and at 30, 60, and $120 \mathrm{~min}$ after reperfusion (Fig. 1).

\section{Surgical Procedure}

Under anesthesia using isoflurane, the animals were tracheotomized. To reduce spontaneous breathing, animals were ventilated mechanically (MK-V100; Muromachi Kikai Co., Ltd., Tokyo, Japan).
The animals were placed in a supine position on a heated pad to maintain the rectal temperature at $37^{\circ} \mathrm{C}$. To monitor arterial blood pressure and allow continuous infusion of Ringer's solution, polyethylene catheters (PE-50, 0.58/0.96-mm internal/external diameter; Becton Dickinson, Sparks, MD) were inserted into the left carotid artery and left jugular vein, respectively. After performing laparotomy by a transverse incision, the ligaments around the liver were dissected to mobilize the left lobe. The left hepatic lobe was exteriorized on a plate specially designed to minimize movements caused by respiration and covered with glass. Surgical procedures were performed using sterile technique. After $60 \mathrm{~min}$ of normal saline continuous infusion, IVM was performed as a pre-ischemia study. Then, hepatic ischemia was induced by clamping the portal triad with a microclip (B. Braun Aesculap Japan Co., Ltd., Tokyo, Japan) for 20 min. IVM was performed at 30,60, and 120 min after reperfusion. Blood samples were taken for analysis of enzyme activities from a catheter placed in the left carotid artery at the same time as IVM. Alanine aminotransferase (ALT) was evaluated as one of the liver enzymes. At 120 min of reperfusion, total body blood was taken for euthanasia. At the end of the experiment, liver tissue was taken for histological examination.

\section{Platelet Preparation}

Platelets were isolated from the whole blood of syngeneic rats and labeled with rhodamine-6G $(50 \mu \mathrm{l} / \mathrm{ml}$ whole blood: R4127; Sigma, St. Louis, MO, USA), as described previously (Massberg et al. 1998). In brief, the collected blood was diluted with buffer after the addition of prostaglandin E1 and rhodamine-6G. After two-cycle centrifugation, fluorescent platelets were suspended in phosphate buffered saline (PBS). In this study, a total of $1 \times 10^{8}$ fluorescencelabeled platelets, approximately $1 \%$ of all circulating platelets in the recipient rat, were injected through the left carotid artery at $5 \mathrm{~min}$ before IVM.

\section{Fluorescence Labeling of KCs}

$\mathrm{KCs}$ were labeled by the liposome entrapment method as described previously (Tamura et al. 2012). Briefly, fluorescent phosphatidylcholine labeled by NBD $\mathrm{C}_{12}$-HPC (2-(12-(7-nitrobenz-2-oxa1,3-diazol-4-yl)amino)dodecanoyl-1-hexadecanoyl-sn-glycero3-phosphocholine: N3787; Molecular Probes, Eugene, USA) was incorporated into liposomes. Sixty min before hepatic ischemia, fluorescent liposomes $(4 \mathrm{ml} / \mathrm{kg}$ ) were administered via the left carotid artery. Then, KCs in the rat livers were stained and clearly delineated in the IVM.

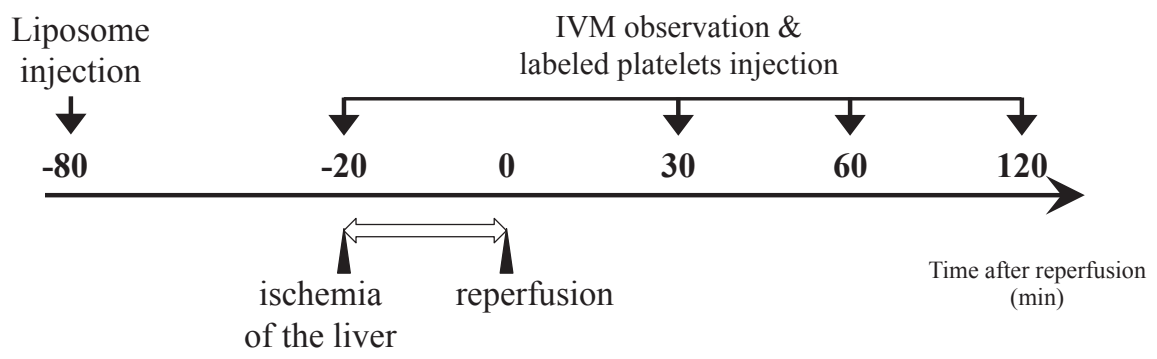

Fig. 1. Experimental design.

In all groups, total warm hepatic ischemia was induced for 20 minutes by clamping the portal triad. A total of $1 \times 10^{8}$ fluorescence-labeled platelets, approximately $1 \%$ of all circulating platelets in the recipient rat, were injected via the left carotid artery $5 \mathrm{~min}$ before IVM (intravital microscopy). 


\section{Intravital Microscopy (IVM)}

IVM was performed using a modified microscope (BX30 FLA-SP; Olympus Co., Tokyo, Japan) with a $100 \mathrm{~W}$ mercury lamp attached to a filter block. The hepatic microcirculation was recorded by means of a CCD camera (C5810; Hamamatsu Photonics, Hamamatsu, Japan) and a digital video recorder (GV-HD700/1; Sony, Tokyo, Japan) for offline analysis. Using objective lenses $(10 \times 0.3$ to $20 \times 0.7$; Olympus Co., Tokyo, Japan), a final magnification from $\times 325$ to $\times 650$ was achieved on the video screen. To assess sinusoidal perfusion, sodium fluorescein $\left(2 \times 10^{-3} \mathrm{M} / \mathrm{kg}, \mathrm{F}-6377\right.$; Sigma, St. Louis, MO) was injected via the jugular catheter. Rhodamine-6G labeled platelets were infused intra-arterially just before ischemia and at 30,60, and $120 \mathrm{~min}$ after reperfusion, and 10 randomly chosen acini were visualized. Quantitative assessment of the microcirculatory parameters was performed offline using WinROOF imaging software (version 5.0; Mitani Shoji, Tokyo, Japan).

\section{Microcirculatory Analysis}

The following parameters were analyzed: (1) the number of adhesions of platelets to KCs in the acini, i.e., platelets firmly attached to $\mathrm{KCs}$ for longer than $20 \mathrm{~s}$ (one acinus is approximately equal to one field [one field = approximately $0.2 \mathrm{~mm}^{2}$ ]); (2) sinusoidal perfusion rate (\%) as an index of microcirculatory disturbance, calculated as the ratio of perfused sinusoids among the sinusoids observed in one acinus after $120 \mathrm{~min}$ of reperfusion (Perfused sinusoids were defined as sinusoid in which the flow of fluorescein microspheres could be viewed without delay during the observation period, and non-perfused sinusoids as those in which the flow of fluorescein microspheres was not seen or noticebly slow.); (3) the number of KCs in the acini.

\section{Histology and Immunohistochemistry}

Liver tissue was obtained before ischemia to assess the degree of steatosis and after 120 min of reperfusion to assess the histological changes due to IR. The samples were fixed with $10 \%$ formalin, and embedded in paraffin. Thin sections $(4 \mu \mathrm{m})$ were prepared and stained with hematoxylin-eosin (HE). Tissue damage was evaluated in 5 randomly selected high-power fields $(\times 200)$. Histological alterations were determined according to the palisade arrangement grade of each acinus as follows: grade 0 , no damage; grade 1 , loss of palisade arrangement in less than half of the sinusoids in the acini; grade 2, loss of palisade arrangement in more than half of the sinusoids in the acini; and grade 3, total loss of palisade arrangement. In addition, the samples were immunolabeled to evaluate histologically the number of $\mathrm{KCs}$ in the acini. The sections were immersed in $0.03 \%$ hydrogen peroxidase to block endogenous peroxidase activity, and then blocked with $2 \%$ bovine serum albumin to reduce background staining. To specifically recognize KCs, mouse anti-rat ED2 antibody (Santa Cruz Biotechnology, Santa Cruz, CA, USA) was used as the primary antibody. The sections were incubated with primary diluted antibodies (1:50) at room temperature for $60 \mathrm{~min}$. Primary antibody reactions were enhanced using horseradish peroxidase EnVision (Dako Japan, Tokyo, Japan). The immunoreaction was visualized with $0.05 \%$ 3,3-diaminobenzidine (DAB) solution. After washing in distilled water, specimens were counterstained with hematoxylin. The number of ED2-positive cells was counted as $\mathrm{KCs}$ in 5 randomly chosen acini.

\section{Biochemical Assays}

As a marker of liver deterioration, serum ALT levels were measured using a Drychem 7000V autoanalyzer (Fuji Film, Tokyo, Japan). In addition, levels of interleukin (IL)-6 in 10\% lysates of liver tissue were measured using commercial ELISA kits (R\&D Systems, Minneapolis, MN, USA).

\section{Fatty Acid Composition of Liver}

An aliquot $(0.1 \mathrm{~g})$ of liver samples snap-frozen by liquid nitrogen was homogenized in $1 \mathrm{~mL}$ of normal saline before ischemia. The fatty acid composition was measured by gas chromatography at SRL, Inc. (Tokyo, Japan). Briefly, total lipids in liver homogenates were extracted according to the Folch procedure, followed by transesterification of fatty acids with boron trifluoride-methanol at $100^{\circ} \mathrm{C}$ for 90 min (Folch et al. 1957). The methylated fatty acids were then extracted with hexane and analyzed using a GC-17A gas chromatograph (Shimadzu Corporation, Kyoto, Japan) and a TC-70 capillary column (GL Sciences Inc., Tokyo, Japan).

\section{Western blotting}

For Western blot analysis, liver protein lysates were prepared, separated by $10 \%$ sodium dodecyl sulfate polyacrylamide gel electrophoresis (SDS-PAGE) and transferred to a nitrocellulose membrane (Millipore, Bedford, MA). Phospho-SAPK/JNK (9251) and totalSAPK/JNK antibodies (9252) (Cell Signaling Technology, Beverly, MA) were used as the primary antibody. Secondary goat anti-rabbit antibody conjugated with horseradish peroxidase was purchased from Zymed Laboratories (San Francisco, CA).

\section{Statistical Analysis}

All data were expressed as mean \pm SEM. The Mann-Whitney test and analysis of variance (ANOVA) were used, followed by Scheffe's test. $P$ values $<0.05$ were considered statistically significant.

\section{Results}

Mild and Moderate Steatotic Liver Models

The rats fed on CDD for 2 weeks histologically had lipid droplet filtration in 10 to $20 \%$ of hepatocytes, so we identified these findings as mild steatosis (Fig. 2). Similarly, we identified the rats fed on CDD for 4 weeks as having moderate steatosis due to the histological finding that there was lipid droplet filtration in 40 to $50 \%$ of their hepatocytes (Fig. 2).

\section{The interaction between KCs and Platelets}

The adhesion of KCs to platelets indicated the interaction between $\mathrm{KCs}$ and platelets. The number of adhesions of $\mathrm{KCs}$ to platelets after reperfusion in sinusoids in the moderate steatosis group was significantly higher than in the normal liver group ( $p<0.01)$, and the number of adhesions in the mild steatosis group was markedly lower than in the normal liver group $(p<0.01)$ (Fig. 3A and B). There was no significant difference between all groups in the number of blood platelets (data not shown).

\section{Sinusoidal Perfusion Rate}

The sinusoidal perfusion rate was one of the indexes 


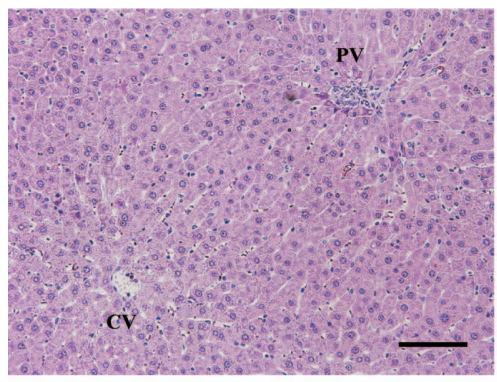

normal liver

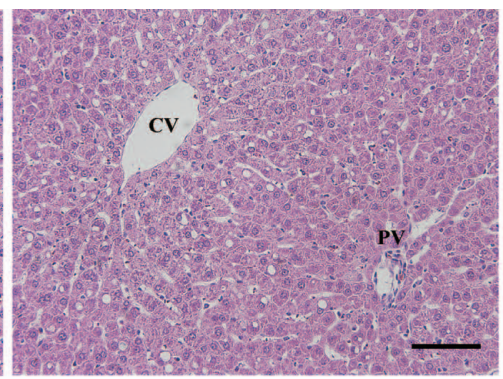

mild steatosis

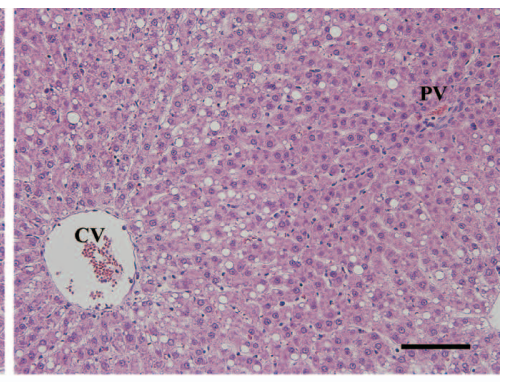

moderate steatosis

Fig. 2. Mild and moderate steatotic liver models (HE stain).

The mild steatosis rats were fed a choline-deficient diet (CDD) for 2 weeks, and the moderate steatosis rats were fed a CDD for 4 weeks. In the mild steatosis group, 10 to $20 \%$ of hepatocytes had depositions of lipid droplets, compared to 40 to $50 \%$ in the moderate steatosis group. PV, portal vein; CV, central vein. Scale bar: $100 \mu \mathrm{m}$.

A

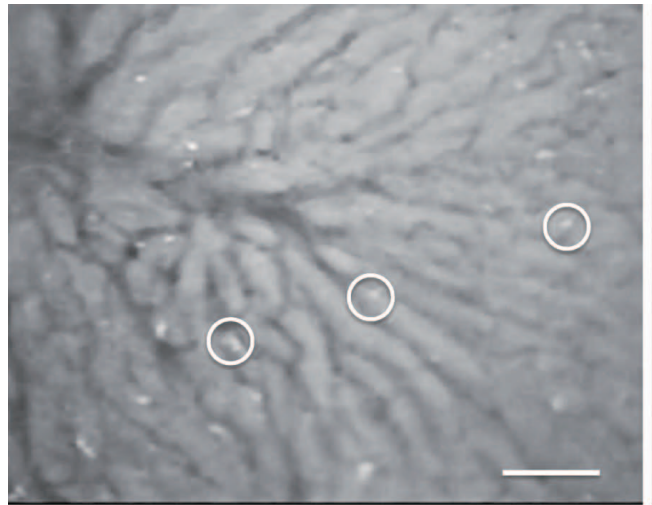

Kupffer cells

B

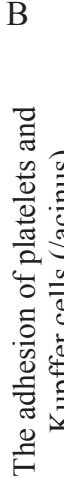

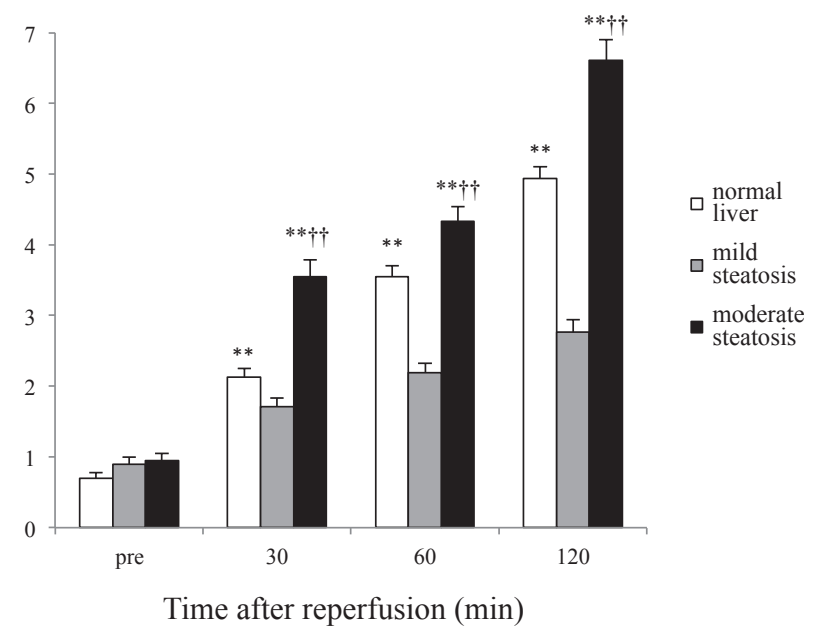

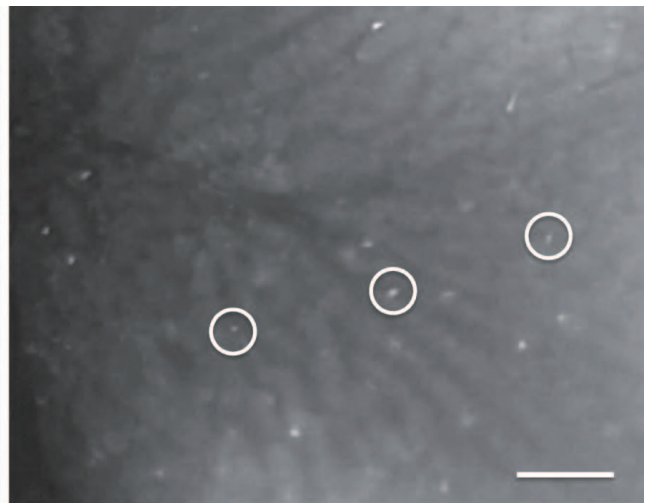

Platelets

$\mathrm{C}$

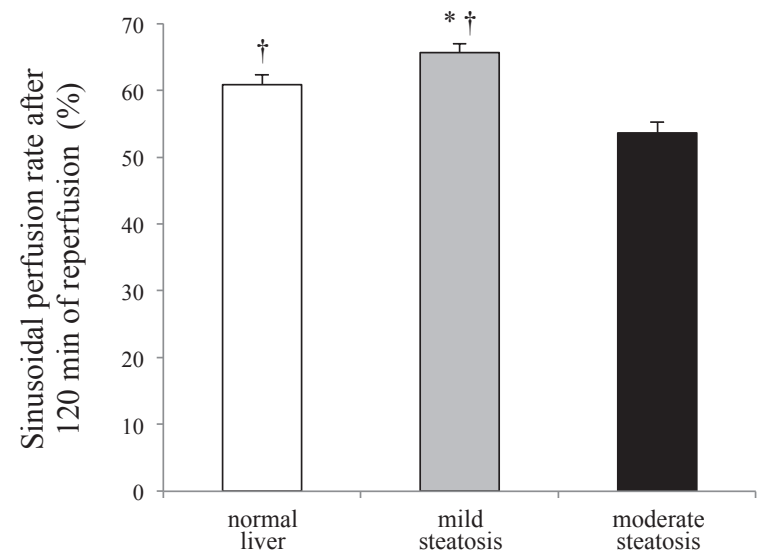

Fig. 3. Intravital microscopy findings.

(A) Video images of Kupffer cells (KCs) and platelets in an acinus $30 \mathrm{~min}$ after reperfusion. The field is approximately $0.2 \mathrm{~mm}^{2}$. The left figure shows the acinus with fluorescently stained $\mathrm{KCs}$, and the right figure, the acinus with fluorescently stained platelets. White circles indicate $\mathrm{KC}$ and platelet adhesion in the same place. Scale bars: $100 \mu \mathrm{m}$. (B) The number of adhesions of platelets and KCs was significantly more in the moderate group than the normal group, and less in the mild steatosis group compared with the normal liver group at $30 \mathrm{~min}$ after reperfusion and concomitant with the duration of reperfusion. Mean $\pm \operatorname{SEM} ; n=6 .{ }^{* *} p<0.01$ vs. the mild steatosis group, $\dagger \dagger p<0.01$ vs. the normal liver group. (C) Sinusoidal perfusion rate. The sinusoidal perfusion rate after $120 \mathrm{~min}$ of reperfusion was significantly higher in the mild steatosis group than the normal liver and moderate steatosis groups. The rate in the normal liver group was higher than in the moderate steatosis group. Mean $\pm \mathrm{SEM} n=6$. ${ }^{*} p<0.05$ vs. the normal liver group, $\dagger p<$ 0.05 vs. the moderate steatosis group. 


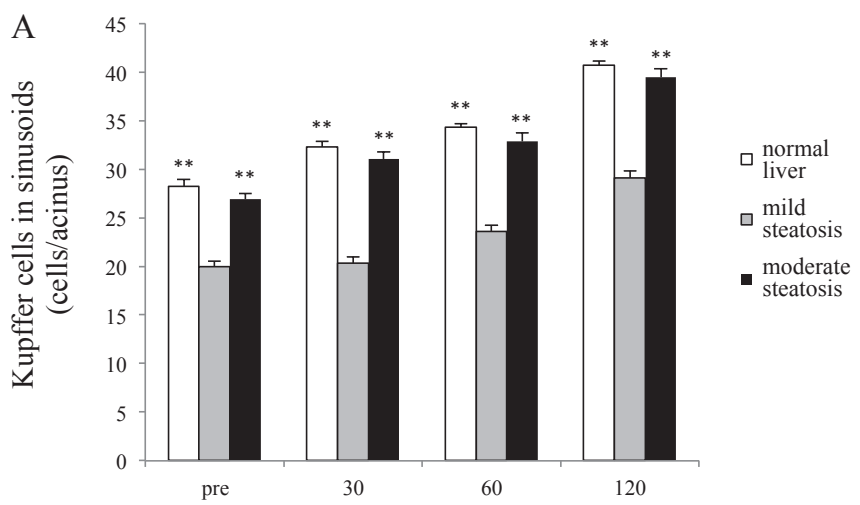

Time after reperfusion ( $\mathrm{min})$
B

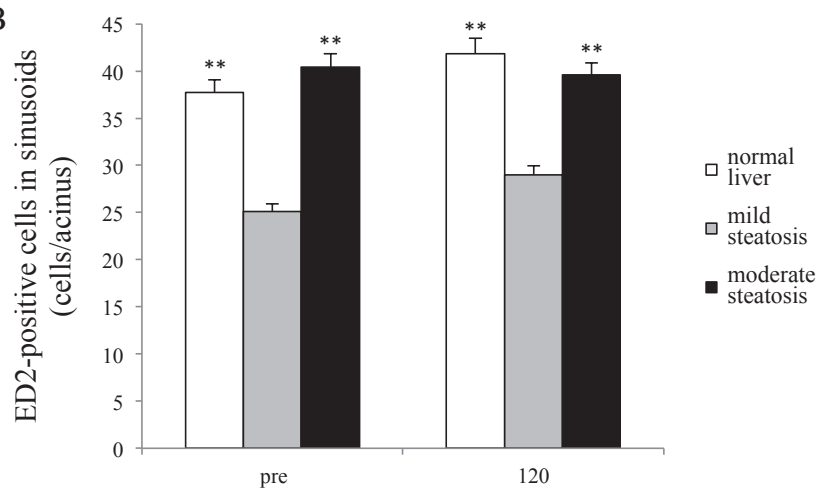

Time after reperfusion (min)

Fig. 4. The number of Kupffer cells in sinusoids.

(A) The number of Kupffer cells in sinusoids observed in the IVM. The numbers of KCs in the normal liver group and the moderate steatosis group were significantly greater than in the mild steatosis group at any point in time before and after IR. On the other hand, there was no difference between the normal liver group and the moderate steatosis group. Mean $\pm \mathrm{SEM} ; n=6 .{ }^{* *} p<0.01$ vs. the mild steatosis group. (B) Immunohistochemical staining of KCs. ED2-positive cells means KCs. Similar to the IVM observation, the numbers of ED2-positive cells in the normal liver group and the moderate steatosis group were significantly more than in the mild steatosis group. Mean $\pm \mathrm{SEM} ; n=6$. $* * p<0.01$ vs. the mild steatosis group.

Table 1. Serum ALT and Liver IL-6 levels.

\begin{tabular}{|c|c|c|c|c|c|}
\hline & After reperfusion & pre ischemia & $30 \mathrm{~min}$ & $60 \min$ & $120 \mathrm{~min}$ \\
\hline \multirow{3}{*}{$\begin{array}{c}\text { Serum ALT } \\
\text { mean } \pm \text { SEM (IU/L) }\end{array}$} & normal liver & $23.3 \pm 0.9$ & $45.2 \pm 6.0$ & $86.5 \pm 12.1$ & $175.0 \pm 29.1$ \\
\hline & mild steatosis & $21.2 \pm 1.9$ & $33.7 \pm 5.0$ & $57.8 \pm 10.5$ & $98.5 \pm 8.1^{*}$ \\
\hline & moderate steatosis & $29.7 \pm 5.6$ & $127.2 \pm 20.6^{* * \dagger \dagger}$ & $219.7 \pm 31.3 * * \dagger \dagger$ & $347.5 \pm 57.2 * * \dagger \dagger$ \\
\hline \multirow{3}{*}{$\begin{array}{c}\text { Liver IL-6 } \\
\text { mean } \pm \text { SEM (ng/g) }\end{array}$} & normal liver & $2.2 \pm 0.04$ & - & - & $4.9 \pm 0.3$ \\
\hline & mild steatosis & $2.2 \pm 0.1$ & - & - & $4.1 \pm 0.3$ \\
\hline & moderate steatosis & $2.3 \pm 0.08$ & - & - & $7.3 \pm 0.1 \dagger$ \\
\hline
\end{tabular}

$* p<0.05$ vs. the normal liver group, ${ }^{* *} p<0.01$ vs. the normal liver group, $\uparrow p<0.05$ vs. the mild steatosis group, $\dagger \dagger p<0.01$ vs. the mild steatosis group.

of hepatic IR injury (Vollmar et al. 1994). After 120 min of reperfusion, the rate of sinusoidal perfusion was significantly higher in the mild steatosis group than in the normal liver group $(p<0.05)$, but was significantly lower in the moderate steatosis group than in the normal liver group $(p<0.05)$ (Fig. 3C).

\section{Number of KCs in Acini}

The numbers of KCs in the normal liver group and the moderate steatosis group were high compared to the mild steatosis group at any point of time before and after IR according to our IVM study ( $p<0.01)$ (Fig. 4A). In addition, the number of KCs was verified immunohistochemically. In immunohistochemical staining, the numbers of ED2-positives in the normal liver group and the moderate steatosis group were greater than in the mild steatosis group $(p<0.01)$ (Fig. 4B). On the other hand, unlike the number of adhesions of KCs to platelets, there was no difference between the normal liver group and the moderate steatosis group in the number of KCs.

\section{Serum ALT and Liver IL-6 Levels}

The serum ALT level was significantly higher in the moderate steatosis group than the normal liver group after 30 min of reperfusion $(p<0.01)$, and was strikingly lower in the mild steatosis group than in the normal liver group after 120 min of reperfusion $(p<0.05)$ (Table 1). IL-6 concentration in the liver tissue showed a tendency similar to the serum ALT levels, however, there was no significant difference except for between the mild steatosis group and the moderate steatosis group ( $p<0.05$, after $120 \mathrm{~min}$ of reperfusion) (Table 1).

\section{Histological Findings}

In the normal liver and moderate steatosis groups, disturbance of the hepatocyte palisade arrangement and sinusoidal narrowing were observed. Lymphocyte infiltration was also observed. In addition, such histological damage to the liver was greater in the moderate steatosis group than in the normal liver group. However, these findings were slight in the mild steatosis group compared with the other 2 


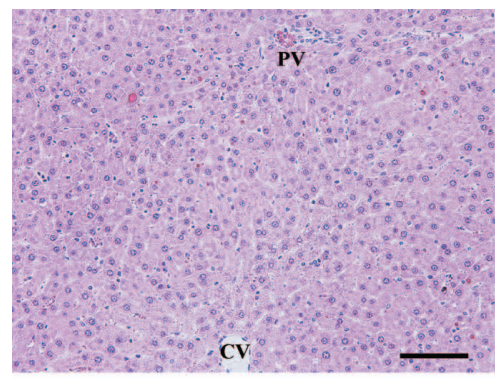

normal liver

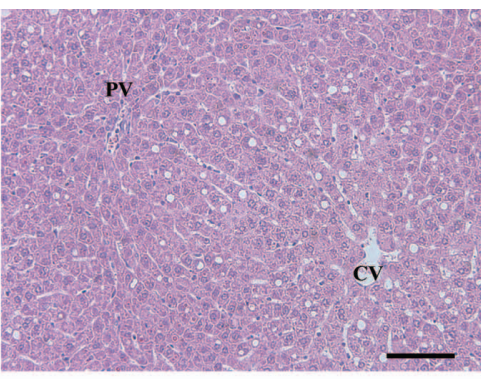

mild steatosis

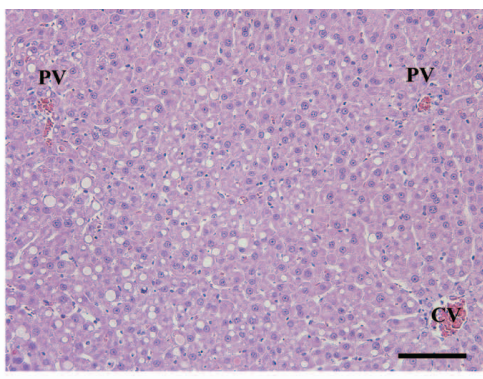

moderate steatosis

Fig. 5. Histological findings after 120 min of reperfusion.

In the normal liver and moderate steatosis, disturbance of the hepatocyte palisade arrangement or sinusoidal narrowing were observed. In addition, such histological changes in the liver were more obvious in moderate steatosis than in the normal liver, however, these findings were slight in mild steatosis compared with the other 2 groups. PV, portal vein; CV, central vein. Scale bar: $100 \mu \mathrm{m}$.

A

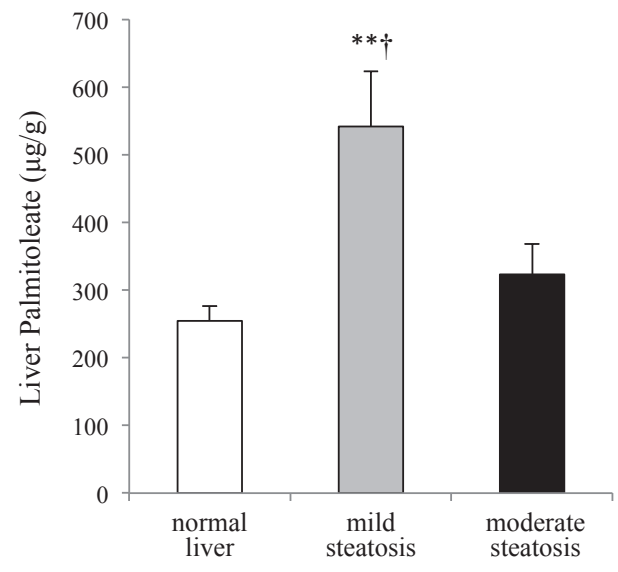

B

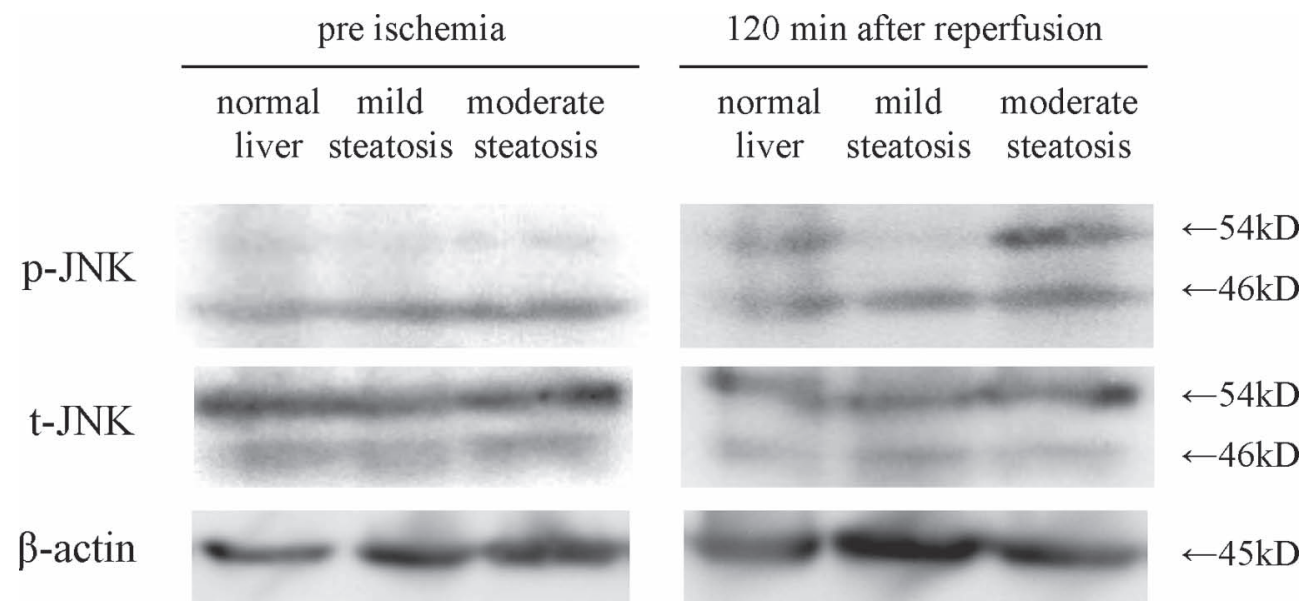

Fig. 6. Liver palmitoleate level and JNK phosphorylation.

(A) Liver palmitoleate levels. In the mild steatosis group, the concentration of palmitoleate in the liver tissue was significantly higher than the other 2 groups. Mean \pm SEM; $n=6$. ${ }^{* *} p<0.01$ vs. the normal liver group, $\dagger p<0.05$ vs. the moderate steatosis group. (B) Phosphorylation of JNK. After $120 \mathrm{~min}$ of reperfusion, phosphorylation of JNK in the normal liver and the moderate steatosis group was observed. On the other hand, phosphorylation of JNK was not detected in the mild steatosis group.

groups (Fig. 5)

\section{The Composition of Palmitoleate in the Liver}

In the mild steatosis group, the concentration of palmi- toleate in the liver tissue was significantly higher than in the other 2 groups ( $p<0.01$ vs. the normal liver group, $p<0.05$ $v s$. the moderate steatosis group) (Fig. 6A). There was no significant difference between the normal liver group and 
the moderate steatosis group (Fig. 6A).

\section{Phosphorylation of JNK}

After 120 min of reperfusion, phosphorylation of JNK in the normal liver group and the moderate steatosis group was observed. On the other hand, phosphorylation of JNK was not detected in the mild steatosis group (Fig. 6B).

\section{Discussion}

It is well known that liver steatosis increases the risk of postoperative complications following major liver resection, since the steatotic liver is particularly vulnerable to IR injury (Selzner and Clavien 2001). Activation of KCs plays a pivotal role in the development of hepatic IR injury (Bremer et al. 1994). Therefore one of the factors that advance IR injury is that KCs are more sensitive to disturbances in microcirculation and dysregulation of cytokine production in the steatotic liver (Teramoto et al. 1993). It has been reported that inactivation of KCs was effective in preventing IR injury in the steatotic liver (Bruns et al. 2011). In our present study, we focused on the interaction between KCs and platelets in IR injury in order to compare the normal liver and the mild and moderate steatotic livers. We evaluated the IR injury by serum ALT levels, liver IL-6 levels, histological findings, and sinusoidal perfusion rate. We demonstrated that the degree of IR injury was not simply proportional to the severity of steatosis.

We have investigated the roles of leukocytes, platelets, and KCs in hepatic IR injury using IVM (Nakano et al. 2008, 2009; Pak et al. 2010; Tamura et al. 2012). We previously showed that KC-platelet interaction such as adhesion induced hepatic IR injury (Tamura et al. 2012, 2013). Moreover, we demonstrated that IR injury in the mild steatotic liver was attenuated compared with the normal liver, and that it resulted from the reduction of KC-platelet interaction due to the decreased number of KCs (Ogawa et al. 2013). In this study, the number of the adhesions of platelets and KCs was inversely correlated with the sinusoidal perfusion rate. This indicated that $\mathrm{KC}$-platelet interaction affects hepatic IR injury through the reduction of sinusoidal perfusion. Compared to the normal liver, moderate steatosis facilitated the adhesion of KCs to platelets, which enhanced the IR injury. The intensity of interaction between KCs and platelets depends on the number of KCs in sinusoids, and the activity of KCs. In addition, the morphological changes such as twisting and narrowing of the sinusoids due to massive fatty depositions may magnify IR injury induced by KC-platelet interaction (Sun et al. 2001; Federico et al. 2010). KCs are more likely to be activated in the steatotic liver (Teramoto et al. 1993; Bruns et al. 2011), and hepatic IR also activates KCs (Zhai et al. 2011). It seems that $\mathrm{KC}$ activation and sinusoidal morphological change in steatosis appeared to increase according to the degree of steatosis. We considered that in moderate steatosis, the KC-platelet interaction increased due to enhancement of $\mathrm{KC}$ activation and sinusoidal morphological change even if the $\mathrm{KC}$ number was equal to that in the normal liver. Aldeguer et al. (2002) hypothesized that liver IL-6 reflects $\mathrm{KC}$ activity as well as the inflammatory response after IR, because KCs are the most potent producers of IL- 6 in the liver. Our data that liver IL-6 levels were high in moderate steatosis compared to the other two models supports those reports.

The present study showed that the number of KCs in the sinusoids was independent of the degree of steatosis. Several studies reported that steatosis altered the number of $\mathrm{KCs}$, however, the influence of fatty change on KCs has not yet been well established (Vetelainen et al. 2007; Shono et al. 2011; Guo et al. 2012). In the present study, the number of KCs in moderate steatosis was greater than in mild steatosis, but was equal to the normal liver. The results were confirmed by both IVM study and immunohistochemical examination. The relationship between the number of KCs and steatosis has not been well investigated yet (Vetelainen et al. 2007; Shono et al. 2011). We focused on the change in the proportions of fatty acids, especially palmitoleate, in the liver, because there were some reports that palmitoleate reduced the numbers of $\mathrm{KCs}$, and decreased the expression of proinflammatory cytokines in the liver (Akazawa et al. 2010; Guo et al. 2012). It was reported that palmitoleate, which is available from dietary sources and produced endogenously by adipocytes, has a protective effect against hepatic lipotoxicity as well as insulin resistance (Matsuzaka et al. 2007; Cao et al. 2008). The progression of steatosis altered the proportions of the various fatty acids in the liver (Sato et al. 2004). Previous investigators reported that KCs critically determine liver inflammatory status (Deng et al. 2009). Thus, it was likely that palmitoleate provided the anti-inflammatory effect on the liver by reducing the number of KCs. Our results demonstrated that the level of palmitoleate in conditions of mild steaotosis was significantly higher than in the normal liver and moderate steatosis. Therefore, it seemed that the difference in the amount of palmitoleate brought about a change in the number of KCs. Palmitoleate also decreases inflammatory signaling through the JNK pathway (Akazawa et al. 2010). JNK, a stressactivated kinase, is induced by various stimuli including hepatic IR (Parola et al. 1998; Theruvath et al. 2008). In this study, phosphorylation of JNK after IR was not observed in mild steatosis due to overexpression of palmitoleate. This result supports the previous report of palmitoleate having a protective effect in liver injury. On the other hand, it is not clear why the proportion of palmitoleate changes with the duration of CDD feeding, so future investigation is necessary.

Our present study suggests that IR injury in the steatotic liver does not intensify parallel to development of steatotic changes. In liver surgery, there is no difference in the resectability between the mild steatotic liver and the normal liver. Furthermore, mild to moderate steatosis sufferers are accepted as marginal donors in liver transplantation (PerezDaga et al. 2006; Briceno et al. 2005). Our results suggest 
that there is more opportunity to utilize donors with mild steatosis. On the other hand, liver with moderate steatosis is not suitable as the graft for liver transplantation. With moderate or worse steatosis, it is necessary to be prudent in the application of liver surgery in order to avoid aggravation of postoperative complications. Also in the steatotic liver, KC-platelet interaction and the number of KCs were strongly associated with hepatic IR injury. Therefore, there is a possibility that the degree of IR injury can be predicted before surgery, if the number of KCs in the liver can be quantified, i.e., by liver biopsy or contrast-enhanced ultrasonography with Sonazoid (Salvatore et al. 2012). For clinical application, further studies will be necessary. Finally, we admit the limitations of studies using the CDD model. It is unclear whether change in $\mathrm{KC}$ numbers in steatosis formation is specific to the CDD model: there may be differences due to the methods of inducing steatosis. However, there is no answer in the literature yet, so investigation using various steatosis models is required.

In conclusion, we demonstrated that IR injury in the steatotic liver was not simply in proportion to the degree of steatosis. Mild steatosis ameliorated IR injury compared to the normal liver, while moderate steatosis aggravated IR injury. Hepatic IR injury was strongly correlated with the degree of interaction between KCs and platelets even in the steatotic liver.

\section{Acknowledgements}

This work was supported in part by Grants-in-Aid from the Ministry of Education, Culture, Sports, Science and Technology of Japan (MEXT).

\section{Conflict of Interest}

The authors declare no conflict of interest.

\section{References}

Adam, R., Reynes, M., Johann, M., Morino, M., Astarcioglu, I., Kafetzis, I., Castaing, D. \& Bismuth, H. (1991) The outcome of steatotic grafts in liver transplantation. Transplant. Proc., 23, 1538-1540.

Akazawa, Y., Cazanave, S., Mott, J.L., Elmi, N., Bronk, S.F., Kohno, S., Charlton, M.R. \& Gores, G.J. (2010) Palmitoleate attenuates palmitate-induced Bim and PUMA up-regulation and hepatocyte lipoapoptosis. J. Hepatol., 52, 586-593.

Aldeguer, X., Debonera, F., Shaked, A., Krasinkas, A.M., Gelman, A.E., Que, X., Zamir, G.A., Hiroyasu, S., Kovalovich, K.K., Taub, R. \& Olthoff, K.M. (2002) Interleukin-6 from intrahepatic cells of bone marrow origin is required for normal murine liver regeneration. Hepatology, 35, 40-48.

Bremer, C., Bradford, B.U., Hunt, K.J., Knecht, K.T., Connor, H.D., Mason, R.P. \& Thurman, R.G. (1994) Role of Kupffer cells in the pathogenesis of hepatic reperfusion injury. Am. J. Physiol., 267, G630-636.

Briceno, J., Padillo, J., Rufian, S., Solorzano, G. \& Pera, C. (2005) Assignment of steatotic livers by the Mayo model for endstage liver disease. Transpl. Int., 18, 577-583.

Bruns, H., Watanpour, I., Gebhard, M.M., Flechtenmacher, C., Galli, U., Schulze-Bergkamen, H., Zorn, M., Buchler, M.W. \& Schemmer, P. (2011) Glycine and taurine equally prevent fatty livers from Kupffer cell-dependent injury: an in vivo microscopy study. Microcirculation, 18, 205-213.

Cao, H., Gerhold, K., Mayers, J.R., Wiest, M.M., Watkins, S.M. \& Hotamisligil, G.S. (2008) Identification of a lipokine, a lipid hormone linking adipose tissue to systemic metabolism. Cell, 134, 933-944.

Delva, E., Camus, Y., Nordlinger, B., Hannoun, L., Parc, R., Deriaz, H., Lienhart, A. \& Huguet, C. (1989) Vascular occlusions for liver resections. Operative management and tolerance to hepatic ischemia: 142 cases. Ann. Surg., 209, 211-218.

Deng, Z.B., Liu, Y., Liu, C., Xiang, X., Wang, J., Cheng, Z., Shah, S.V., Zhang, S., Zhang, L., Zhuang, X., Michalek, S., Grizzle, W.E. \& Zhang, H.G. (2009) Immature myeloid cells induced by a high-fat diet contribute to liver inflammation. Hepatology, 50, 1412-1420.

Federico, A., D’Aiuto, E., Borriello, F., Barra, G., Gravina, A.G., Romano, M. \& De Palma, R. (2010) Fat: a matter of disturbance for the immune system. World J. Gastroenterol., 16, $4762-4772$.

Folch, J., Lees, M. \& Sloane Stanley, G.H. (1957) A simple method for the isolation and purification of total lipides from animal tissues. J. Biol. Chem., 226, 497-509.

Guo, X., Li, H., Xu, H., Halim, V., Zhang, W., Wang, H., Ong, K.T., Woo, S.L., Walzem, R.L., Mashek, D.G., Dong, H., Lu, F., Wei, L., Huo, Y. \& Wu, C. (2012) Palmitoleate induces hepatic steatosis but suppresses liver inflammatory response in mice. PLoS One, 7, e39286.

Hayashi, M., Tokunaga, Y., Fujita, T., Tanaka, K., Yamaoka, Y. \& Ozawa, K. (1993) The effects of cold preservation on steatotic graft viability in rat liver transplantation. Transplantation, 56, 282-287.

Hui, A.M., Kawasaki, S., Makuuchi, M., Nakayama, J., Ikegami, T. \& Miyagawa, S. (1994) Liver injury following normothermic ischemia in steatotic rat liver. Hepatology, 20, 1287-1293.

Ijaz, S., Yang, W., Winslet, M.C. \& Seifalian, A.M. (2003) Impairment of hepatic microcirculation in fatty liver. Microcirculation, 10, 447-456.

Massberg, S., Eisenmenger, S., Enders, G., Krombach, F. \& Messmer, K. (1998) Quantitative analysis of small intestinal microcirculation in the mouse. Res. Exp. Med (Berl)., 198, 23-35.

Matsuzaka, T., Shimano, H., Yahagi, N., Kato, T., Atsumi, A., Yamamoto, T., Inoue, N., Ishikawa, M., Okada, S., Ishigaki, N., Iwasaki, H., Iwasaki, Y., Karasawa, T., Kumadaki, S., Matsui, T., et al. (2007) Crucial role of a long-chain fatty acid elongase, Elovl6, in obesity-induced insulin resistance. Nat. Med., 13, 1193-1202.

McCormack, L., Petrowsky, H., Jochum, W., Furrer, K. \& Clavien, P.A. (2007) Hepatic steatosis is a risk factor for postoperative complications after major hepatectomy: a matched casecontrol study. Ann. Surg., 245, 923-930.

Nakano, Y., Kondo, T., Matsuo, R., Hashimoto, I., Kawasaki, T., Kohno, K., Myronovych, A., Tadano, S., Hisakura, K., Ikeda, O., Watanabe, M., Murata, S., Fukunaga, K. \& Ohkohchi, N. (2008) Platelet dynamics in the early phase of postischemic liver in vivo. J. Surg. Res., 149, 192-198.

Nakano, Y., Kondo, T., Matsuo, R., Murata, S., Fukunaga, K. \& Ohkohchi, N. (2009) Prevention of leukocyte activation by the neutrophil elastase inhibitor, sivelestat, in the hepatic microcirculation after ischemia-reperfusion. J. Surg. Res., 155, 311-317.

Ogawa, K., Kondo, T., Tamura, T., Matsumura, H., Fukunaga, K., Oda, T. \& Ohkohchi, N. (2013) Influence of Kupffer cells and platelets on ischemia-reperfusion injury in mild steatotic liver. World J. Gastroenterol., 19, 1396-1404.

Pak, S., Kondo, T., Nakano, Y., Murata, S., Fukunaga, K., Oda, T., Sasaki, R. \& Ohkohchi, N. (2010) Platelet adhesion in the sinusoid caused hepatic injury by neutrophils after hepatic ischemia reperfusion. Platelets, 21, 282-288.

Parola, M., Robino, G., Marra, F., Pinzani, M., Bellomo, G., 
Leonarduzzi, G., Chiarugi, P., Camandola, S., Poli, G., Waeg, G., Gentilini, P. \& Dianzani, M.U. (1998) HNE interacts directly with JNK isoforms in human hepatic stellate cells. $J$. Clin. Invest., 102, 1942-1950.

Perez-Daga, J.A., Santoyo, J., Suarez, M.A., Fernandez-Aguilar, J.A., Ramirez, C., Rodriguez-Canete, A., Aranda, J.M., Sanchez-Perez, B., Montiel, C., Palomo, D., Ruiz, M. \& Mate, A. (2006) Influence of degree of hepatic steatosis on graft function and postoperative complications of liver transplantation. Transplant. Proc., 38, 2468-2470.

Salvatore, V., Borghi, A. \& Piscaglia, F. (2012) Contrast-enhanced ultrasound for liver imaging: recent advances. Curr. Pharm. Des., 18, 2236-2252.

Sato, H., Mohamed, T., Goto, A., Oikawa, S. \& Kurosawa, T. (2004) Fatty acid profiles in relation to triglyceride level in the liver of dairy cows. J. Vet. Med. Sci., 66, 85-87.

Selzner, M. \& Clavien, P.A. (2001) Fatty liver in liver transplantation and surgery. Semin. Liver Dis., 21, 105-113.

Shono, S., Habu, Y., Nakashima, M., Sato, A., Nakashima, H., Miyazaki, H., Kinoshita, M., Tsumatori, G., Shinomiya, N. \& Seki, S. (2011) The immunologic outcome of enhanced function of mouse liver lymphocytes and Kupffer cells by high-fat and high-cholesterol diet. Shock, 36, 484-493.

Sun, C.K., Zhang, X.Y., Zimmermann, A., Davis, G. \& Wheatley, A.M. (2001) Effect of ischemia-reperfusion injury on the microcirculation of the steatotic liver of the Zucker rat. Transplantation, 72, 1625-1631.

Tamura, T., Kondo, T., Ogawa, K., Fukunaga, K. \& Ohkohchi, N.
(2013) Protective effect of heme oxygenase-1 on hepatic ischemia-reperfusion injury through inhibition of platelet adhesion to the sinusoids. J. Gastroenterol. Hepatol., 28, 700-706.

Tamura, T., Kondo, T., Pak, S., Nakano, Y., Murata, S., Fukunaga, K. \& Ohkohchi, N. (2012) Interaction between Kupffer cells and platelets in the early period of hepatic ischemia-reperfusion injury: an in vivo study. J. Surg. Res., 178, 443-451.

Teramoto, K., Bowers, J.L., Kruskal, J.B. \& Clouse, M.E. (1993) Hepatic microcirculatory changes after reperfusion in fatty and normal liver transplantation in the rat. Transplantation, 56, 1076-1082.

Theruvath, T.P., Snoddy, M.C., Zhong, Z. \& Lemasters, J.J. (2008) Mitochondrial permeability transition in liver ischemia and reperfusion: role of c-Jun N-terminal kinase 2. Transplantation, 85, 1500-1504.

Vetelainen, R., van Vliet, A. \& van Gulik, T.M. (2007) Essential pathogenic and metabolic differences in steatosis induced by choline or methione-choline deficient diets in a rat model. $J$. Gastroenterol. Hepatol., 22, 1526-1533.

Vollmar, B., Glasz, J., Leiderer, R., Post, S. \& Menger, M.D. (1994) Hepatic microcirculatory perfusion failure is a determinant of liver dysfunction in warm ischemia-reperfusion. Am. J. Pathol., 145, 1421-1431.

Zhai, Y., Busuttil, R.W. \& Kupiec-Weglinski, J.W. (2011) Liver ischemia and reperfusion injury: new insights into mechanisms of innate-adaptive immune-mediated tissue inflammation. Am. J. Transplant., 11, 1563-1569. 